\title{
Challenges in the regulatory use of water-accommodated fractions for assessing complex substances
}

\author{
James R. Wheeler ${ }^{1}$, Delina Lyon², Carolina Di Paolo ${ }^{1}$, Albania Grosso ${ }^{3}$ and Mark Crane ${ }^{3 *}$ (D)
}

\begin{abstract}
The use of the water-accommodated fraction (WAF) approach for the preparation of exposure systems of complex substances such as petroleum products has been a standard way to perform aquatic toxicity tests on these substances for over 30 years. In this Commentary, we briefly describe the historical development, rationale, and guidance for the use and reporting of the WAF approach to assess complex substances. We then discuss two case studies, with coal tar pitch and kerosene/jet fuel, which illustrate challenges from regulatory authorities in Europe and the United States when using the WAF approach. We describe how the WAF approach is the only currently known method for testing the toxicity of the whole of a complex substance, even when some of its constituents remain unknown; it accounts for differences in the solubility of the constituents within a complex substance; and use of loading rates to describe any toxic effects is a unifying concept that allows direct comparison with releases of readily soluble substances in hazard assessment and chemical classification.
\end{abstract}

Keywords: Water-accommodated fraction, WAF, UVCB

\section{Background}

The use of the water-accommodated fraction (WAF) approach for the preparation of exposure systems of complex substances such as petroleum products has been a standard way to perform aquatic toxicity tests on these substances for over 30 years [18]. However, there have been recurrent misinterpretations by various regulatory bodies of the data generated for these types of tests on substances of "Unknown or Variable Composition, Complex reaction products and Biological materials" (UVCBs). In the past, the United States Environmental Protection Agency (USEPA) has also questioned the use of WAF data in the High Production Volume (HPV) Challenge Program [35].

In this Commentary, we briefly describe the historical development, rationale, and guidance for the use and

\footnotetext{
*Correspondence: mark.crane@ag-hera.com

${ }^{3}$ AG-HERA, 23 London Street, Faringdon SN7 7AG, Oxfordshire, UK

Full list of author information is available at the end of the article
}

reporting of the WAF approach to assess UVCBs. We then discuss two cases in which reported WAF results have been challenged by regulatory agencies: a recently concluded legal case in which European regulatory authorities were found to have misinterpreted results from WAF testing of coal tar, and a separate case in which the USEPA rejected WAF data in their assessment of kerosene/jet fuel. Clarifying the WAF methodology and data interpretation is important because of the very large number of substances that have been classified as UVCBs in both Europe and the United States. For example, Sauer et al. [30] report that approximately $20 \%$ of substances registered under REACH [10] have been declared as UVCBs [16], and approximately $25 \%$ of substances listed on the USEPA Toxic Substances Control Act Inventory are identified as UVCBs $[37,38]$. Therefore, the proper performance of WAF studies, consistent application of the dosing metric, and appropriate reporting and use of these data are critically important for clear and transparent regulatory processes. 


\section{What is a water-accommodated fraction (WAF) and how is it prepared?}

Girling et al. [9, 18], and ECETOC [13] extensively explain why common procedures followed by standard test methods for measuring aquatic toxicity are difficult to apply to UVCBs such as petroleum products, attributing these challenges mainly to their relative insolubility, high volatility, and multi-constituent nature. These methodological issues have been addressed by the OECD Guidance Document on aquatic toxicity testing of difficult substances and mixtures [27] by recommending testing approaches which involve assessment of only their "water-soluble fraction" (WSF) or their WAF. By nature of their preparation and their use in aquatic toxicity testing, the constituents in a WAF will not be present in the same concentration as in the original test substance, as the constituents will only reach a saturation limit in the WAF proportional to their water solubility and their concentration in the test substance. A WAF is prepared by gently stirring the test medium with a known loading of the test substance for an amount of time pre-determined to allow saturation (Fig. 1). After ceasing stirring and allowing the phases to settle, the droplet-free water fraction is then siphoned or otherwise removed for use as the testing medium. The key references cited above argue that the results of such tests should be expressed as a "loading rate", which is the ratio of test substance to aqueous medium used in preparation

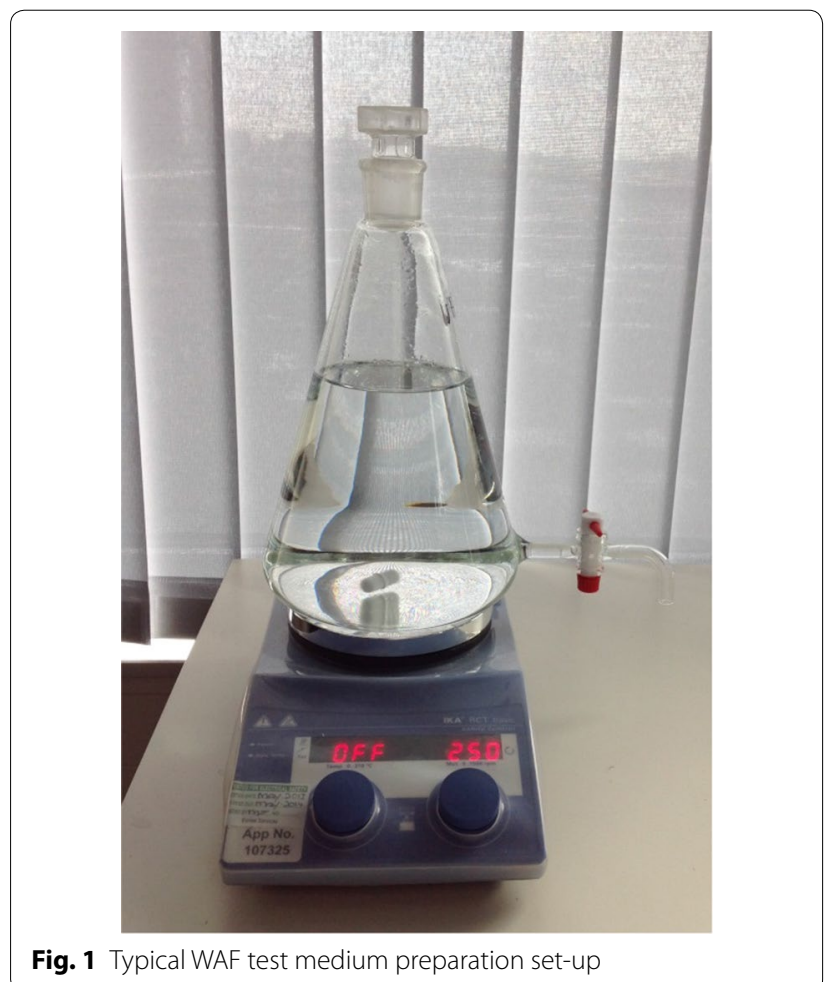

of the aquatic toxicity test medium. The loading rate is then expressed as either a Lethal Loading (LL) or an Effective Loading (EL) when reporting toxicity test results for, respectively, lethal or sublethal effects in acute tests. Typically, the No Observable Effect Loading Rate (NOELR) and Lowest Observable Effect Loading Rate (LOELR) are used for long-term chronic studies.

Special preparation techniques are important because the solubility of UVCB substances (or rather the constituents therein) is a complex issue [4]. Which constituents are dissolved in the water phase, and the proportions of each of these, depend not only on the initial loading, but also on interactions amongst the constituents and extrinsic factors such as temperature, salinity, and mixing duration which may affect constituent solubilities. Therefore, a whole host of processes involving solid-liquid interactions, co-solvent effects, volatility, and degradation require an environmentally relevant and integrated description. In addition, there is the inescapable fact that the composition of UVCBs is variable and often not fully known, so standard approaches to exposure characterisation cannot be reliably applied.

As a result of this, in WAF tests the exposure expression is based on the nominal concentration used to prepare the WAF and not on any measure of the dissolved material that the test organisms are exposed to in the test system. This concept is of particular significance for UVCBs as the exposure expression represents the whole substance and not just the bioavailable fraction that is presented to the test organism.

\section{WAF good experimental practices}

The approach described above is standard in regulatory aquatic toxicity testing (with the highest test concentration being determined by the test substance's solubility in test media, or by a limit level) in order to avoid non-chemical or physical effects of particles that may otherwise overestimate toxicity. The procedure is also supported by the fact that effects that are not inherent to the investigated chemical toxicity are not considered relevant to likely realistic exposures in the environment.

Figure 2 illustrates the process for good practice when preparing WAF test media for aquatic toxicity tests:

A. A preparation trial is performed to demonstrate the mixing time required to achieve equilibrium of the dissolved phase (which determines the duration of the stir period). Sealed vessels should be considered to minimise losses if some constituents are known to be volatile. For regulatory testing, each treatment level is preferably prepared as an individual WAF and is not serially diluted from a higher loaded WAF. Serial dilution is not recommended as, due to the wide range of 

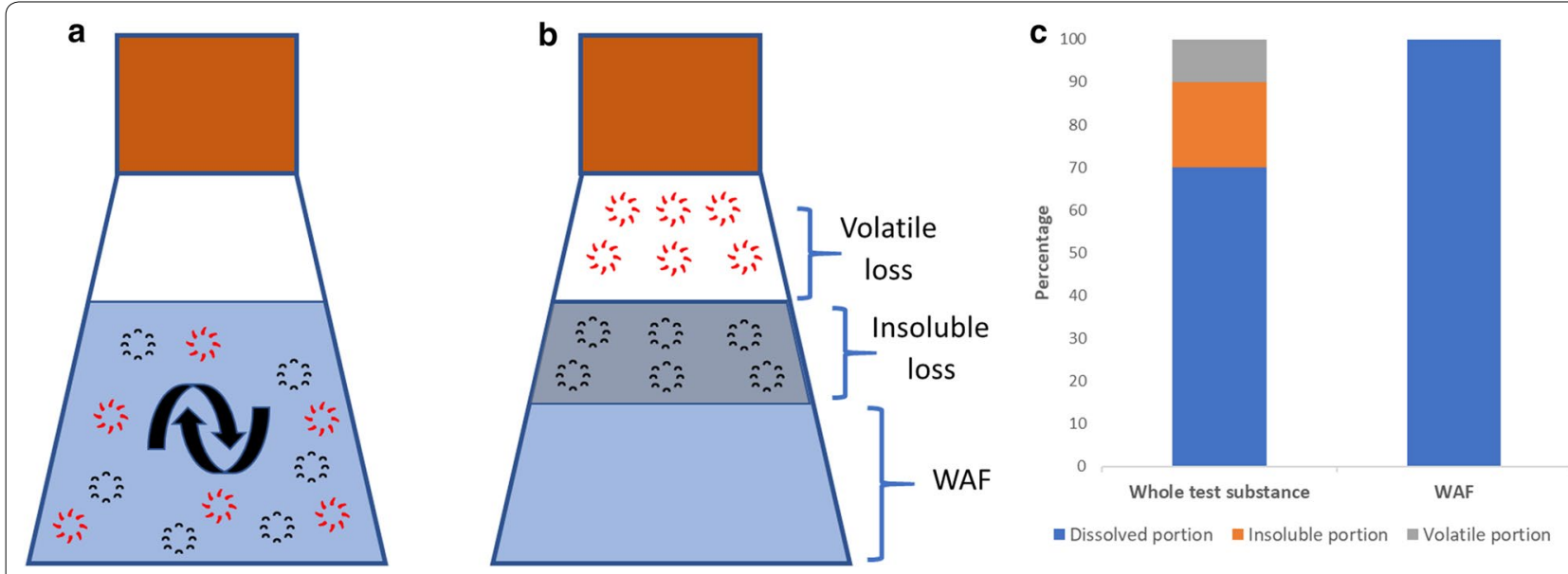

Fig. 2 WAF test medium preparation (see text for details)

physicochemical properties of the constituents, there may not be a linear correlation between the aqueous concentration of the individual constituents and the loading rate of the test substance [32]. However, serial dilution of one WAF loading rate may be necessary if the WAF is highly toxic and the accuracy to measure small amounts of test material (i.e. lower than $1 \mathrm{mg} / \mathrm{L}$ ) introduces greater error than serial dilution [27]. Efforts are made to standardise mixing energies across individual WAFs, for example by measuring vortex depth. The above steps are intended to maximise the dissolution of components into test media and ensure consistency of preparation. Chemical analysis (or a surrogate measure such as total organic carbon) is important to determine if acceptable loading of the WAF has been achieved [27], and for assuring that the ideal preparation time to reach equilibrium is employed in the definitive study.

B. The resulting WAF mixture is left to settle for a suitable period to ensure separation of the aqueous phases and/or sedimentation of particles. The test exposure medium is then taken via siphon or other means to exclude the non-dissolved fraction. In some cases, it may be necessary to perform a further separation step (e.g. filtration or centrifugation) to remove any suspended undissolved emulsified components-the resulting solution is then referred to as a water-soluble fraction (WSF). In both WAF and WSF regulatory tests, undissolved material/constituents should be removed whenever possible.

C. Therefore, the exposure concentration in a subsequent aquatic toxicity test represents only the bioavailable dissolved fraction of the test material.
It is important that the procedures above are standardised and described in as much detail as possible in study reports. This allows for the best possible repetition of procedures in following studies (tests in other species, etc.) or clarifying work. Even relatively small differences in preparation may impact the resulting WAF composition and hence reduce the direct comparability across studies.

ECETOC [13] notes that UVCB WAF preparation often produces test solutions with a composition that is quite different to that of the substance itself. This is because when the solubility of different constituents varies the composition of the test medium will depend on the method of preparation. This is for the reasons described above and has more recently been indirectly demonstrated in in vitro systems in which DMSO extraction of petroleum substances was shown to select for certain polycyclic aromatic compounds, with different dilutions leading to different chemical profiles and bioavailabilities [24]. Chemical analysis of WAF solutions during toxicity testing can therefore mechanistically aid interpretation of the data. Chemical analysis may also help to (i) determine the relationship between the toxic end point and the actual exposure concentrations, (ii) assess the stability of the exposure over the test period and, in some cases, (iii) identify the active constituent. This is in addition to the importance of analysis to demonstrate that the duration of mixing during preparation of the WAF was sufficient to maximise the concentration of dissolved constituents (see above). However, no current guidance recommends that these measurements of individual constituent concentrations should be used to report toxicity results. It is also important to note that for such complex and variable substances chemical analysis cannot fully support 
mixture toxicity assessment (e.g. possible antagonistic or synergistic effects) as captured by bioanalytical tools.

The recommended approach to report the entire WAF loading rate rather than analytically determined constituents is supported by guidance for the environmental hazard classification of chemical substances. For example, the United Nations Globally Harmonized System of Classification and Labelling of Chemicals [34] as well as OECD guidance documents on the classification of chemicals which are Hazardous for the Aquatic Environment $[26,27]$ state that the loading level may be used directly in the classification criteria. Under REACH guidance [15] test data obtained with WAFs apply to the multi-constituent substance as an entity. The exposure is generally expressed as the 'loading rate' (mass-to-volume ratio of the substance to medium) used to prepare the WAF, although the measured concentration of test substance in the WAF can also be used. Use of either of these types of measurement in chemical risk assessment is not supported because their comparison with a Predicted Environmental Concentration is for a different entity entirely. This comparison is therefore meaningless except in certain specific applications such as oil spill risk assessment. Instead, constituent-based approaches are more meaningful for chemical risk assessment.

\section{Disadvantages of the WAF approach}

The WAF approach is a pragmatic solution to the testing and assessment of complex substances. However, it has limitations compared to approaches that are applicable to simpler substances. From an experimental point of view there is greater uncertainty as to the actual chemical exposure within the test system. Further, even minor differences in preparation technique can alter the composition of the WAF. Both these uncertainties can hamper extrapolation across studies within and amongst different laboratories. Analytical verifications of selected components can be helpful in this regard and are recommended [27]. However, for the reasons described above, they do not represent the whole test substance or necessarily even the behaviour of all components that make it into the WAF or WSF exposure medium. This can limit our ability to interpret any observed effects, or lack thereof, in relation to demonstrated exposure. By extension, this limits the use of WAF data for quantitative risk assessment as described above for REACH assessments.

\section{Potential WAF improvements and alternatives}

There are alternatives to WAF approaches. In 2000, the Organisation for Economic Cooperation and Development (OECD) first published Guidance Document 23 on aqueous-phase aquatic toxicity testing of difficult-to-test chemicals (now revised and updated [27]). OECD [27] outlines several alternative dosing techniques for sparingly soluble substances that could be applied to UVCBs. These include generator systems, saturator columns, and passive dosing. Generator and saturator systems may not be ideal for UVCBs since elution of the various constituents may vary over time. Therefore, the eluent will not be a constant reflection of the soluble portion of the test item over time. Passive dosing systems apply a biocompatible polymer pre-loaded with the test substance which acts as a partitioning donor that controls exposure concentrations throughout the test, although currently available passive dosing polymers are unlikely to cover the wide range of constituent $\log \mathrm{K}_{\mathrm{ow}}$ values found in complex mixtures of polar and non-polar substances. Despite these other possible dosing options, in a section of the Guidance Document on multi-constituent substances (which now includes UVCBs in the updated version) the use of WAF and loading rates is still recommended, and this guidance is also referred to in Guidance Document 27 on classification of chemicals [26]. OECD [27] recommends the following process for aquatic toxicity testing of UVCBs:

1. Analytically determine the composition of the UVCB so that its identity can be confirmed and its constituents tracked throughout testing.

2. If a UVCB is only partially soluble in water then prepare a WAF for each individual loading rate, according to a set of described standard methods.

3. Report the results as a median lethal loading (LL50), median effective loading (EL50), or No Observable Effect Loading Rate (NOELR), which are determined following the same statistical methods as would be used to determine LC50, EC50, and NOEC values, respectively.

\section{WAF as the method of choice for petroleum products}

The simplicity and wide applicability of WAFs and loading rates mean they have been used extensively in aquatic toxicity testing, especially for petroleum products, since the seminal Girling et al. [18] paper. For example, WAF testing has been performed on algae (e.g. $[8,23])$, crustaceans (e.g. $[3,6])$, gastropod and bivalve molluscs (e.g. $[25,33])$, and fish (e.g. [5, 28]), in both freshwaters (e.g. [31]) and saltwaters (e.g. [7]). Singer et al. [32] describe the extensive work undertaken by the Chemical Response to Oil Spills Ecological Research Forum (CROSERF) to develop standardised approaches for performing aquatic toxicity tests with WAFs, including analytical verification of test concentrations, sealing test vessels to minimise volatilisation, and the need to prepare individual treatment loading rates instead of serially diluting from a stock solution (as above). 
In summary, WAF testing of UVCBs and the expression of test results as loading rates has the following advantages:

1. It is the only currently known method for testing the toxicity of the whole of a complex substance, even when some of its constituents remain unknown;

2. It accounts for differences in the solubility of the constituents within a complex substance; and

3. Use of loading rates to describe any toxic effects is a unifying concept that allows direct comparison with releases of readily soluble substances in hazard assessment and classification.

\section{Regulatory challenges to the WAF approach}

However, despite this understanding of the theoretical advantages, and successful practical use, of WAF testing over three decades, the approach has recently been challenged and frequently dismissed by regulatory authorities. We provide two examples of this below: one for coal tar regulation in Europe and one for kerosene jet fuel regulation in the United States.

\section{Example 1: coal tar pitch, high temperature (CTPHT)}

Coal tar pitch, high temperature (CTPHT; CAS \# 6599693-2, EC number 266-028-2) is a UVCB substance containing polycyclic aromatic hydrocarbons (PAHs). It is mainly used to produce refractories and electrode binders for the aluminium and steel industry, with minor uses as clay targets, coating for corrosion protection, keroseneresistant airfield applications, road construction, roofing and briquetting [19]. CTPHT is produced or imported into the EU at a volume of more than 100 thousand tonnes per year.

Several WAF tests have been performed with CTPHT, as summarised in ECHA's Committee for Risk Assessment (RAC) Opinion [29]. These include different WAF test solution preparation methods (direct addition without filtration, direct addition with supernatant after the solid material was siphoned out of solution, and dilution of the saturated stock) in the absence of ultraviolet irradiation in acute toxicity tests of fish and invertebrates. The direct addition method (with and without filtration) produced CTPHT LL50 values between 100 and $1000 \mathrm{mg} / \mathrm{L}$ for all species. The dilution method produced an LL50 of $>1000 \mathrm{mg} / \mathrm{L}$ for the fish Oryzias latipes (other species were not tested).

WAF toxicity studies with algae (Desmodesmus subspicatus) and daphnids (Daphnia magna) were also performed (ground material, slow stirred, and settled) in the absence of ultraviolet radiation and with no analytical determination of test media. Both tests produced EL50 values $>100 \mathrm{mg} / \mathrm{L}$.

A semi-static chronic study with D. magna was performed using crushed and filtered material pumped through a column at a nominal loading of $100 \mathrm{mg} / \mathrm{L}$. The test solution was analysed for individual PAH concentrations. No significant effects on daphnid mortality and reproduction were observed in this test.

Additional information is available in the RAC opinion paper [29] on the solubility of pulverised CTPHT. This indicated that the WSF of single PAHs was measured at concentrations far below individual water solubilities and was consistent with analytical measurements within the D. magna chronic study.

Despite the WAF results described above, in September 2010, a proposal was submitted to the European Chemicals Agency (ECHA) for a classification for CTPHT as Aquatic Acute 1 (H400), and Aquatic Chronic 1 (H410) under the CLP Regulation [11]. This classification was upheld by ECHA's Risk Assessment Committee [29]. In its consideration of environmental hazards the $\mathrm{RAC}$ agreed that CTPHT is a UVCB substance and therefore (i) is very difficult to classify on the basis of its individual constituents; (ii) not all the constituents can be analysed when diluted in water; and (iii) different CTPHT constituents influence each other's solubility in the water phase and so the composition in water will not be the same at different loadings. The RAC concluded that the WAF approach is most appropriate for classifying CTPHT, citing OECD guidance described earlier in this Commentary.

However, the RAC also pointed out that all the toxicity data for CTPHT from the WAF studies were obtained in the absence of ultraviolet irradiation, although several PAHs are known to be phototoxic. In addition, they were concerned that chemical analytical data were only measured at a loading of $100 \mathrm{mg} / \mathrm{L}$ in a chronic Daphnia study and that this prevented comparison between the dissolved $\mathrm{PAH}$ concentrations at different loadings and the toxicity data obtained for the individual PAHs. As a result of these concerns an alternative approach for the environmental classification of CTPHT was taken by the Netherlands competent authority and the RAC, in which CTPHT was considered a "mixture", with classification based on the summation of a subset of constituents. The lowest available EC50 or LC50 values for 16 individual polyaromatic hydrocarbons (PAHs) that are also CTPHT constituents were collated and combined with degradability and bioaccumulation data to produce an individual classification for each. Multiplying (M) factors were then used according to the EU CLP [17] to multiply the weight-based percentage of each constituent in CTPHT according to its toxicity. The contributions of all classifiable Aquatic Acute 1 (H400) substances and Aquatic Chronic 1 (H410) 
substances were then summed to produce an overall estimate of the contribution of these substances' toxicity to the overall acute or chronic toxicity of CTPHT.

The results of this process for both acute and chronic toxicity are shown in Table 1 . The estimated contribution of the individual PAHs to the overall acute toxicity of CTPHT is $14521 \%$ and the contribution to overall chronic toxicity is $2169 \%$. These values are considerably higher than the 25\% limit in the CLP regulation so RAC [29] proposed to classify CTPHT as Aquatic Acute 1 and Chronic 1 , and this classification was adopted by the European Commission in October 2013.

There are two main issues with the process followed by the European Commission to classify CTPHT as Aquatic Acute 1 and Chronic 1:

1. It was inconsistent with the provisions of $\mathrm{REACH}$ and EU CLP [12] guidance documents regarding WAF test results interpretation and use in support of classification of UVCBs; and

2. It was inconsistent with the European Commission's own technical guidance on dealing with UVCBs. Indeed, even if the Commission had considered the substance to be a "mixture" and not a UVCB, the tiered classification approach for mixtures would first consider test results for the mixture itself rather than of its constituents.

After 18 companies decided to appeal against the classification of CTPHT as toxic for the aquatic environment the European General Court ruled in October 2015 [19] that the Commission had committed a manifest error of assessment. The Court ruling stated that "neither the RAC's opinion on CTPHT nor the background document contains any reasoning which demonstrates that, in assuming that all of the PAHs present in that substance dissolve in the water phase and are available to aquatic organisms, account was taken of the low water solubility of CTPHT" and "by assuming that all of those PAHs dissolve in water, the Commission therefore, in essence, based the classification in question on the assumption that $9.2 \%$ of CTPHT could dissolve in water. However, ... such a value is not realistic, given that the maximum rate is $0.0014 \%$.

The European Commission immediately appealed this decision, but lost that appeal in a judgement in November 2017 [21] based on legal opinion provided by the Advocate General in September 2017 [20]. Costs were awarded against the Commission at a level finally agreed by the Court in April 2020 [22].

CTPHT has now been classified for the environment in the EU as Chronic 4 (the lowest, "safety net" classification available), which is more consistent with WAF data on its solubility and aquatic toxicity.
Even though regulators acknowledged the applicability of WAF data, there was a reluctance to use the data. This may be related to the complexity of UVCB risk assessment in general. WAF testing of this UVCB was always both the most technically appropriate and regulatorily compliant method, subject only to the relatively minor technical question of whether such testing should be conducted with or without UV radiation.

\section{Example 2: kerosene/jet fuel}

Kerosene and jet fuels are complex petroleum-derived UVCBs that are used as blending components for finished fuels such as aviation turbine fuels (jet fuels), No. 1-K kerosene (for heating and illumination), and No. 1 grades of fuel oil, diesel fuel, and gas turbine oil. In September 2010 the American Petroleum Institute submitted a kerosene/jet fuel Category Assessment Document to USEPA as part of the High Production Volume (HPV) Challenge Program [1].

The HPV Challenge Program is a voluntary initiative to develop and make publicly available screening-level health and environmental effects information on chemicals manufactured in or imported into the United States in quantities greater than one million pounds (454 tonnes) per year. In the Challenge Program, producers and importers of HPV chemicals voluntarily sponsor chemicals and identify and assess the adequacy of existing toxicity data, conduct new testing if adequate data do not exist, and make both new and existing data and information available to the public.

Several WAF tests were reported in the submission to the HPV Challenge Program for kerosene/jet fuel [1].

Two sets of aquatic acute toxicity studies (fish, invertebrate, and algae) were reported for hydrodesulfurised kerosene (CAS \# 64742-81-0), sweetened kerosene (CAS \# 91770-15-9), and hydrocracked naphtha (CAS \# 10131680-7). Fish tests with rainbow trout produced similar LL50 values of $10 \mathrm{mg} / \mathrm{L}$ and $100 \mathrm{mg} / \mathrm{L}$. Tests with aquatic invertebrates resulted in some of the lowest toxicity endpoints for the three species but also some of the most variable, with values between 1.4 to $89 \mathrm{mg} / \mathrm{L}$ for the three substances. The toxicity to algae of these substances was reported as 5.0 and $30 \mathrm{mg} / \mathrm{L}$.

The API Testing Group also commissioned a 21-day reproduction test with $D$. magna exposed to hydrodesulfurised kerosene. The study used independent WAF preparations for the exposure solutions, and the total dissolved hydrocarbons in each WAF treatment were measured by gas chromatography. When expressed as WAF loading rate, kerosene produced EL50 values of $0.89 \mathrm{mg} / \mathrm{L}$ for reproduction and $0.81 \mathrm{mg} / \mathrm{L}$ for immobilisation, with LOELR and NOELR values of $1.2 \mathrm{mg} / \mathrm{L}$ and $0.48 \mathrm{mg} / \mathrm{L}$, respectively, for all endpoints. When expressed as mean 


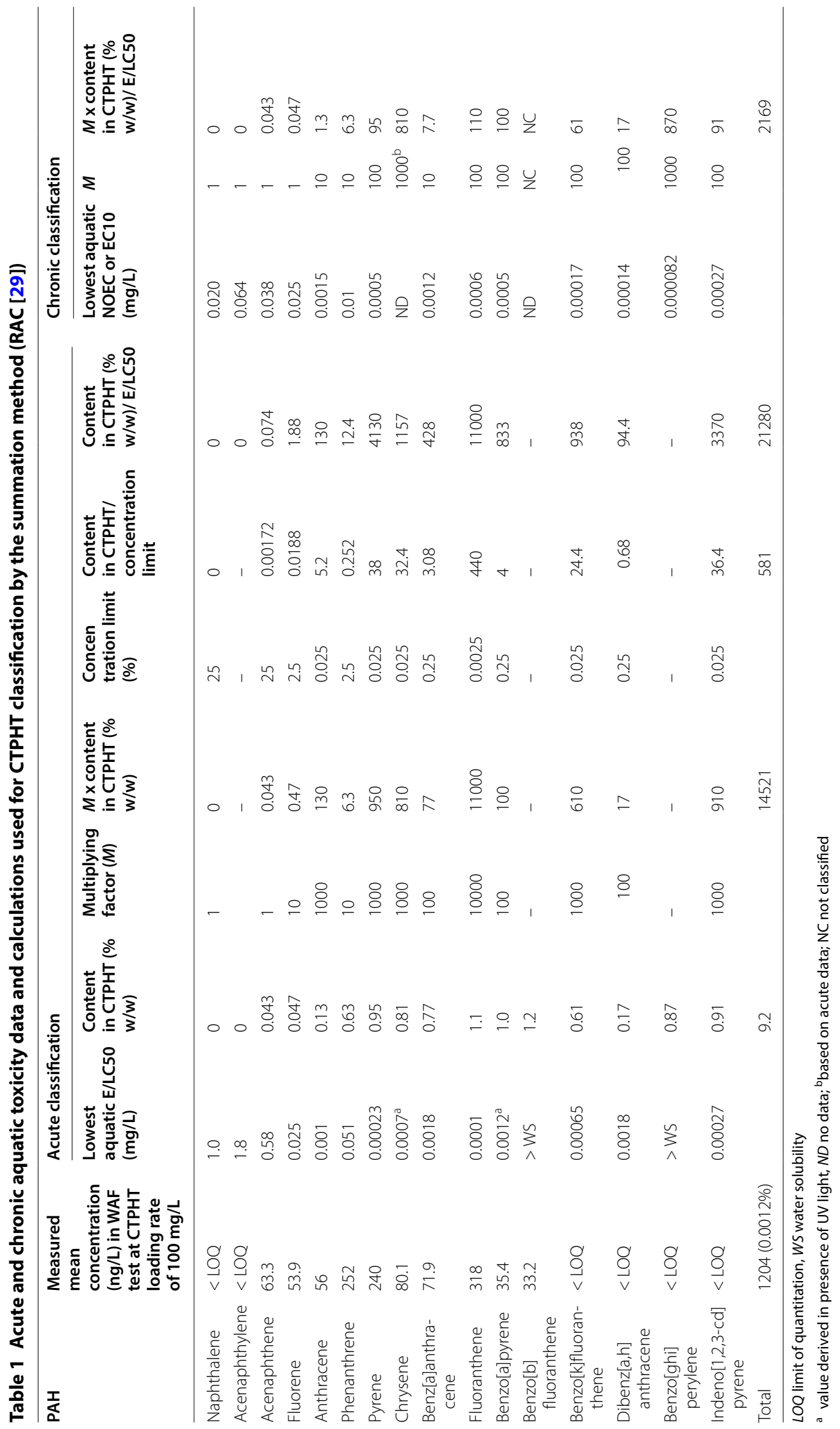


measured concentrations of dissolved hydrocarbons the EC50 values were $0.16 \mathrm{mg} / \mathrm{L}$ for reproduction and $0.15 \mathrm{mg} / \mathrm{L}$ for immobilisation and the LOEC and NOEC were $0.23 \mathrm{mg} / \mathrm{L}$ and $0.092 \mathrm{mg} / \mathrm{L}$, respectively, for all endpoints.

Despite submission of the WAF results described above, USEPA declared in their Hazard Characterization Document [36] that: "For aquatic toxicity, data submitted for the category member, hydrodesulfurized kerosene (petroleum) (CASRN 64742-81-0), were considered inadequate because the results were reported based on nominal loading rates, not measured concentrations. Similarly, data submitted for CASRN 91770-15-9 and 101316-80-7 are not adequate." As a result of this, USEPA proceeded to characterise kerosene/jet fuel aquatic hazard based on read across from measured concentrations of selected hydrocarbons and not on the basis of loading rates from WAF tests for either hydrodesulfurised kerosene or the two supporting substances (sweetened kerosene and hydrocracked naphtha).

In their response to this USEPA decision, the API [2] reiterated the view that results for UVCBs should be expressed as lethal loadings rather than lethal/effect concentrations. This is because the loading rate reflects the composition and chemistry of the substance and implicitly accounts for dissolution and volatilisation of individual hydrocarbon constituents. In contrast to this, they viewed results expressed as measured concentrations of the fraction of the substance in solution as of little value because it prevents extrapolation to spill situations where the only relevant measures of concentration will be the amount of product spilled and the volume of the receiving environment (i.e. the loading rate).

The two case studies presented in this Commentary demonstrate a continuing unease within some regulatory authorities to use WAF loadings directly for hazard assessment. Indeed, this is reflected in more recent examples experienced by authors of this Commentary in which authorities have increasingly requested that study results are expressed in terms of mean measured concentrations rather than nominal WAF loadings. Depending on the substance this can have ramifications for classification and labelling of substances, leading to increased transport costs or further restrictions that are not truly commensurate with the intrinsic hazard of the whole substance. This is not an issue affecting only a small number of substances: 16968 of 68091 substances currently listed on the non-confidential TSCA Inventory are UVCBs [38], and in early 2017 around $21 \%$ of the substances registered under $\mathrm{REACH}$, amounting to more than 2500 chemicals, were UVCBs [16].

\section{Conclusions}

The use of the WAF is the most technically coherent approach to evaluate the toxicological properties of UVCB substances for classification and hazard assessment purposes. This is enshrined in guidance documents published by the OECD and ECHA in support of the implementation of REACH and CLP legislations. However, the examples provided above and frequent recent requests to industry for aquatic toxicity endpoints to be expressed in terms of measured values suggest that some regulatory authorities do not appear to agree with the current state of toxicological evaluation of UVCBs and have questioned the validity of the WAF approach for the expression of toxicity values. This contradicts established international guidance and ECHA legislation in which the substance as a whole, i.e. the UVCB itself and not its individual constituents, is the entity that requires evaluation regarding classification purposes.

There has been clarity about the use of WAF loading rates for hazard/risk assessment and CLP for almost 30 years, since Girling et al. [18] stated the following:

"A major benefit which accrues from expressing [WAF] results in terms of loading rates is that the data are of real value when carrying out a hazard assessment. Results expressed simply in terms of a measured concentration of the fraction of the product in solution are of little value since it will be virtually impossible to extrapolate to a field situation in which the only relevant measures of concentration will be the amount of product spilled and the volume of the receiving environment i.e. the loading rate.

For the purposes of classification and labelling, loading rates allow products which are poorly soluble mixtures and those that are readily soluble pure substances or mixtures to be compared on an equal basis. Criteria for classifying substances as 'dangerous for the environment'... are related to potential concentrations of materials in the environment. Clearly the concentration (or loading rate) of a material in the environment will not only be a function of its relative water solubility but also the quantity entering the environment and the dispersive mechanisms that operate. Consequently, a water soluble product found to be non-toxic at a concentration of $100 \mathrm{mg} / \mathrm{l}$ in a laboratory toxicity test should receive the same classification as a poorly soluble product found to be non-toxic at a loading rate of $100 \mathrm{mg} / \mathrm{l} \mathrm{in}$ a similar test."

Use of WAF is also one of the only ways to test and report on the "unknown" fraction of UVCBs that either are not, or cannot, be analytically measured. The European Chemicals Agency [14] summarises how the WAF 
approach, as described by the OECD [27], might be used under REACH for UVCBs that are highly insoluble in water. Their advice is that test data obtained with WAFs apply to the multi-constituent substance as a whole entity and can be used to provide a justification that aquatic toxicity is unlikely to occur, based on an assessment that includes all constituents that are dissolved or present as a stable dispersion or emulsion.

We hope this commentary highlights the practical and regulatory issues associated with the testing of UVCBs and underscores the importance of WAF approaches for appropriate environmental hazard evaluation and classification purposes.

\begin{abstract}
Abbreviations
API: American Petroleum Institute; CAS: Chemical abstracts service; CLP: Classification, labelling, and packaging; CONCAWE: Conservation of Clean Air and Water in Europe; CROSERF: Chemical response to oil spills research forum; CTPHT: Coal tar pitch, high temperature; ECETOC: European centre for ecotoxicology and toxicology of chemicals; EC: European Commission; ECHA: European Chemicals Agency; EC50: Median effective concentration; EL: Effective loading; EL50: Median effective loading; EU: European Union; GD: Guidance document; HPV: High production volume; LC50: Median lethal concentration; LL: Lethal loading; LL50: Median lethal loading; LOELR: Lowest observable effect loading rate; LOQ: Limit of quantitation; M: Multiplying factor; NC: Not classified; ND: No data; NOEC: No observed effect concentration; NOELR: No observable effect loading rate; OECD: Organisation for Economic Cooperation and Development; PAH: Polyaromatic hydrocarbon; RAC: Risk Assessment Committee; UNECE: United Nations Economic Commission for Europe; USEPA: United States Environmental Protection Agency; UV: Ultraviolet; UVCB: Unknown or variable composition, complex reaction products, and biological materials; WAF: Wateraccommodated fraction; WS: Water solubility; WSF: Water-soluble fraction.
\end{abstract}

\section{Acknowledgements}

Not applicable.

\section{Authors' contributions}

Each author made substantial contributions to the conception, analysis, and interpretation of data and assisted in drafting the work. Each author approved the submitted version (and any substantially modified version that involves the author's contribution to the study) and agrees both to be personally accountable for the author's own contributions and to ensure that questions related to the accuracy or integrity of any part of the work, even ones in which the author was not personally involved, are appropriately investigated, resolved, and the resolution documented in the literature. All authors read and approved the final manuscript.

\section{Funding}

MC and AG received no funding to write this article. Authors who work for Shell received their normal salary.

\section{Availability of data and materials}

The datasets used and/or analysed during the current study are available from the corresponding author on reasonable request.

Ethics approval and consent to participate Not applicable.

\section{Consent for publication}

Not applicable.

\section{Competing interests}

MC and AG are partners in a consultancy (AG-HERA) which works on projects for companies that use WAF test results. The other three authors work for a company (Shell) that submits WAF test results to regulatory authorities.

\section{Author details}

${ }^{1}$ Shell Health, Shell International B.V, The Hague, The Netherlands. ${ }^{2}$ Shell Health, Shell Oil Company, Houston, TX, USA. ${ }^{3}$ AG-HERA, 23 London Street, Faringdon SN7 7AG, Oxfordshire, UK.

Received: 3 October 2020 Accepted: 3 November 2020

Published online: 30 November 2020

\section{References}

1. API (2010) Kerosene/Jet Fuel Category Assessment Document submitted to the US EPA by the American Petroleum Institute Petroleum HPV Testing Group Consortium Registration \# 1100997 September 21, 2010. https ://petroleumhpv.org/ /media/PetroleumHPV/Documents/2010_sept2 1_kerosene_jet\%20fuel\%20CAD\%20final.pdf. Accessed 23 Sep 2020.

2. API (2013) Response to EPA's Hazard Characterization of the Kerosene/Jet Fuel Category. The American Petroleum Institute Petroleum HPV Testing Group June 17, 2013. https://petroleumhpv.org/petroleum-substances -and-categories/ /media/9E3D6B235DA649BDA7847D8E981AF4A4.ashx Accessed 23 Sep 2020.

3. Asadi MA, Khoiruddin AD (2017) pH effects in the acute toxicity study of the crude oil-WAF (water accommodated fraction) in the whiteleg shrimp, Litopenaeus vannamei. AACL Bioflux. 10:1248-56. https://www.biofl ux.com.ro/docs/2017.1248-1256.pdf. Accessed 23 Sep 2020.

4. Birch H, Redman AD, Letinski DJ, Lyon DY, Mayer P (2019) Determining the water solubility of difficult-to-test substances: a tutorial review. Anal Chim Acta 1086:16-28. https://doi.org/10.1016/j.aca.2019.07.034

5. Blenkinsopp S, Sergy G, Doe K, Wohlgeschaffen G, Li K, Fingas M (1997) A method of exposing fish to water-accommodated fractions of oil. Int Oil Spill Confer Proceed 1:941-942. https://doi. org/10.7901/2169-3358-1997-1-941

6. Blinova I, Kanarbik L, Sihmäe M, Kahru A (2016) Toxicity of water accommodated fractions of Estonian shale fuel oils to aquatic organisms. Arch Environ Contam Toxicol 70:383-391. https://doi.org/10.1007/s0024 4-015-0242-8

7. Brakstad OG, Faksness L-G (2000) Biodegradation of water-accommodated fractions and dispersed oil in the seawater column. Soc Petrol Eng Paper Number. https://doi.org/10.2118/61466-MS

8. Chao M, Shen X, Lun F, Shen A, Yuan Q (2012) Toxicity of fuel oil water accommodated fractions on two marine microalgae, Skeletonema costatum and Chlorela spp. Bull Environ Contam Toxicol 88:712-716. https:// doi.org/10.1007/s00128-012-0525-y

9. CONCAWE (1993) Ecotoxicological testing of petroleum products: Test methodology. Report No. 92/56, Brussels, Belgium. https://www.conca we.eu/wp-content/uploads/2017/01/rpt_92-56ocr-2004-01720-01-e.pdf. Accessed 23 Sep 2020.

10. EC (2006) Regulation (EC) No 1907/2006 of the European Parliament and of the Council on the Registration, Evaluation, Authorisation and Restriction of Chemicals (REACH). European Commission, Brussels, Belgium. https ://eur-lex.europa.eu/legal-content/EN/TXT/PDF/?uri=CELEX:02006R1907 -20140410\&from=EN. Accessed 23 Sep 2020.

11. EC (2008) Regulation (EC) No 1272/2008 on the classification, labelling and packaging of substances and mixtures (CLP Regulation). European Commission, Brussels. https://eur-lex.europa.eu/legal-content/EN/TXT/ PDF/?uri=CELEX:32008R1272\&from=EN. Accessed 23 Sep 2020.

12. EC (2017) Guidance on the Application of the CLP Criteria Guidance to Regulation (EC) No 1272/2008 on classification, labelling and packaging (CLP) of substances and mixtures Version 5.0 July 2017. European Commission, Brussels. https://echa.europa.eu/documents/10162/23036412/ clp_en.pdf/58b5dc6d-ac2a-4910-9702-e9e1f5051cc5. Accessed 23 Sep 2020.

13. ECETOC (1996) Aquatic toxicity testing of sparingly soluble, volatile and unstable substances. Monograph No. 26, Brussels. https://www.eceto c.org/wp-content/uploads/2014/08/MON-026.pdf. Accessed 23 Sep 2020.

14. ECHA (2012) How to bring your registration dossier in compliance with REACH - Tips and Hints Part 1. Hints and Tips on physicochemical, environmental and human health related endpoints 27 September, 2012, 15:00 - 17:00 Helsinki Time. Environmental endpoints. Aquatic Toxicity. Speaker: Eva Valkovicova. https://echa.europa.eu/documents/10162 /22840145/04b_webinar20120927_hints+and+tips+on+aquatic+toxic 
ity+adaptations_compliance_pt1_en.pdf/a6451db4-8998-45c1-b67158f1eebf548f. Accessed 23 Sep 2020.

15. ECHA (2013) Guidance on Information Requirements and Chemical Safety Assessment Chapter R.7b: Endpoint specific guidance. European Chemicals Agency, Helsinki, Finland. https://doi.org/10.2823/84188. https:// www.echa.europa.eu/documents/10162/13632/information_requiremen ts_r7b_en.pdf.

16. ECHA (2017) European Chemicals Agency. Read-Across Assessment Framework. Considerations on multi-constituent substances and UVCBs. ECHA-17-R-04-EN; March 2017. https://echa.europa.eu/documents/10162 /13630/raaf_uvcb_report_en.pdf/3f79684d-07a5-e439-16c3-d2c8da96a3 16. Accessed 23 Sep 2020

17. EU (2008) Regulation (EC) No 1272/2008 of the European Parliament and of the Council of 16 December 2008 on classification, labelling and packaging of substances and mixtures, amending and repealing Directives 67/548/EEC and 1999/45/EC, and amending Regulation (EC) No 1907/2006. OJ L 353:1-1355. https://eur-lex.europa.eu/legal-content/EN/ TXT/PDF/?uri=CELEX:32008R1272\&from=EN. Accessed 23 Sep 2020.

18. Girling AE, Markarian RK, Bennett D (1992) Aquatic toxicity testing of oil products-some recommendations. Chemosphere 24:1469-1472. https:// doi.org/10.1016/0045-6535(92)90268-V

19. InfoCuria Case Law (2015) Judgment of the General Court (Fifth Chamber) 7 October 2015. Environment and protection of human health—Classification of pitch, coal tar, high-temperature, in the categories of acute aquatic toxicity and chronic aquatic toxicity — Regulation (EC) No 1907/2006 and Regulation (EC) No 1272/2008—Manifest error of assessment-Classification of a substance on the basis of its constituents. ECLI:EU:T:2015:767. https://curia .europa.eu/juris/document/document.jsftext=\&docid=169267\&pagel ndex $=0 \&$ doclang $=e n \&$ mode $=\mid s t \& d i r=\& o c c=$ first $\&$ part $=1 \& c i d=28322$. Accessed 23 Sep 2020

20. InfoCuria Case Law (2017a) Opinion of Advocate General Bobek delivered on 7 September 2017. Case C-691/15 P. ECLI:EU:C:2017:646. https://curia .europa.eu/juris/document/document.jff;jsessionid=1764450A19CBF58 41CBCE6D9098DE42C?text=\&docid=194120\&pagelndex=0\&docla $\mathrm{ng}=$ en\&mode $=\mid \mathrm{st} \& \mathrm{di}=\& \mathrm{occ}=$ first\&part $=1 \& \mathrm{cid}=189418$. Accessed 23 Sep 2020.

21. InfoCuria Case Law (2017b) Judgment of the Court (Sixth Chamber) 22 November 2017. Appeal-Environment-Regulation (EC) No 1272/2008-Classification, labelling and packaging of certain substances and mixtures—Regulation (EU) No 944/2013 —Classification of pitch, coal tar, high-temperature - Categories of acute aquatic toxicity $(\mathrm{H} 40 \mathrm{O})$ and chronic aquatic toxicity ( $\mathrm{H} 410)$ —Duty to act diligently — Manifest error of assessment. ECLI:EU:C:2017:882. https://curia.europa.eu/juris/document/docum ent.jsf;jsessionid=1764450A19CBF5841CBCE6D9098DE42C?text=\&docid $=197001 \&$ pagelndex $=0 \&$ doclang $=$ en\&mode $=\mid \mathrm{st} \&$ dir $=\& o c c=$ first \&part=1\&cid=189418. Accessed 23 Sep 2020.

22. InforCuria Case Law (2020) Order of the Court (Tenth Chamber) 22 April 2020 (Taxation of costs) in Case C-691/15 P-DEP. ECLI:EU:C:2020:284. https://curia.europa.eu/juris/document/document.jsf?text=\&docid $=225921$ \&pagelndex $=0 \&$ doclang $=$ en $\&$ mode $=$ req\&dir $=\& o c c=$ first \&part=1\&cid=189418. Accessed 23 Sep 2020.

23. Jiang Z, Huang Y, Xu X, Liao Y, Shou L, Liu J, Chen Q, Zeng J (2010) Advance in the toxic effects of petroleum water accommodated fraction on marine plankton. Acta Ecol Sin 30:8-15. https://doi.org/10.1016/j.chnae s.2009.12.002

24. Luo YS, Ferguson KC, Rusyn I, Chiu WA (2020) In vitro bioavailability of the hydrocarbon fractions of dimethyl sulfoxide extracts of petroleum substances. Toxicol Sci 174:168-177. https://doi.org/10.1093/toxsci/kfaa007

25. MacFarlane GR, Reid DJ, Esguerra CA (2004) Sublethal behavioural effects of the water accommodated fractions of crude oil to gastropod molluscs. Bull Environ Contam Toxicol 72(5):1025-1031. https://doi.org/10.1007/ s00128-004-0346-8

26. OECD (2001) Guidance Document on the use of the Harmonised System for the Classification of Chemicals which are Hazardous for the Aquatic Environment. Series on Testing and Assessment No. 27. ENV/JM/ MONO(2001)8, Organisation for Economic Cooperation and Development, Paris, France. https://www.oecd-library.org/docserver/97892 64078444-en.pdf?expires $=1600867308 \& i d=i d \& a c c n a m e=$ guest\&check sum $=7191363884356300$ B05BDCE6E11A45B1. Accessed 23 Sep 2020

27. OECD (2019) Guidance Document on Aqueous-Phase Aquatic Toxicity Testing of Difficult Test Chemicals. Series on Testing and Assessment No. 23 (Second Edition). ENV/JM/MONO(2000)6/REV1, Organisation for Economic Cooperation and Development, Paris, France. https://www. oecd-ilibrary.org/docserver/0ed2f88e-en.pdf?expires $=1600867360$ \&id=id\&accname $=$ guest\&checksum =3EEC2DC68F21D6CBB5AD21D0C 89A3CE3. Accessed 23 Sep 2020.

28. Perrichon P, Le Menach K, Akcha F, Cachot J, Budzinski H, Bustamante $P$ (2016) Toxicity assessment of water-accommodated fractions from two different oils using a zebrafish (Danio rerio) embryo-larval bioassay with a multilevel approach. Sci Total Environ 568:952-966. https://doi. org/10.1016/j.scitotenv.2016.04.186

29. RAC (2011) Annex 1 Background document to the Opinion proposing harmonised classification and labelling at Community level of pitch, coal tar, high temp. (CTPHT) ECHA/RAC/CLH-O-0000001380-85-03/A1 Adopted 21 November 2011. European Chemicals Agency Committee for Risk Assessment, Helsinki. https://echa.europa.eu/documents/10162/ f8d9c3df-6000-8ab7-9d8b-bb1e66694e03. Accessed 23 Sep 2020.

30. Sauer UG, Barter RA, Becker RA, Benfenati E, Berggren E, Hubesch B, Hollnagel HM, Inawaka K, Keene AM, Mayer P, Plotzke K, Skoglund R, Albert O (2019) 21 st Century Approaches for Evaluating Exposures, Biological Activity, and Risks of Complex Substances: Report of an ICCA-LRI Workshop Coorganised with the European Commission's Joint Research Centre. https:// Iri.americanchemistry.com/ICCA-LRI-2019-WS-Full-Report-20191218.pdf. Accessed 23 Sep 2020.

31. Schein A, Scott JA, Mos L, Hodson PV (2009) Oil dispersion increases the apparent bioavailability and toxicity of diesel to rainbow trout (Oncorhynchus mykiss). Environ Toxicol Chem 28:595-602. https://doi. org/10.1897/08-315.1

32. Singer MM, Aurand D, Bragin GE, Clark JR, Coelho GM, Sowby ML, Tjeerdema RS (2000) Standardization of the preparation and quantitation of water-accommodated fractions of petroleum for toxicity testing. Mar Pollut Bull 40:1007-1016. https://doi.org/10.1016/S0025-326X(00)00045-X

33. Solé M, Buet A, Ortiz L, Maynou F, Bayona JP, Albaigés J (2007) Bioaccumulation and biochemical responses in mussels exposed to the water-accommodated fraction of the Prestige fuel oil. Scientia Marina 71:373-382. https://doi.org/10.3989/scimar.2007.71n2373

34. UNECE. (2020) Globally Harmonized System of Classification and Labelling of Chemicals (GHS). United Nations Economic Commission for Europe https://www.unece.org/trans/danger/publi/ghs/ghs_welcome_e.html Accessed 23 Sep 2020.

35. USEPA. (2000) Data collection and development on High Production Volume (HPV) chemicals. Federal Register 2000;65(248)81686-98. https ://www.govinfo.gov/content/pkg/FR-2000-12-26/pdf/00-32498.pdf. Accessed 23 Sep 2020.

36. USEPA. (2011) Hazard Characterization Document: Screening-Level Hazard Characterization Kerosene/Jet Fuel Category. United States Environmental Protection Agency. https://petroleumhpv.org/ /media/PetroleumHPV/ Documents/Category_Kerosene_Jet\%20Fuel_March_2011.pdf. Accessed 23 Sep 2020.

37. USEPA. (2015) Toxic Substances Control Act inventory representation for chemical substances of unknown or variable composition, complex reaction products and biological materials: UVCB substances. United States Environmental Protection Agency. https://www.epa.gov/sites/production /files/2015-05/documents/uvcb.pdf. Accessed 23 Sep 2020.

38. USEPA. (2020) Non-confidential Toxic Substances Control Act inventory. United States Environmental Protection Agency. https://www.epa.gov/ tsca-inventory/how-access-tsca-inventory. Accessed 23 Sep 2020.

\section{Publisher's Note}

Springer Nature remains neutral with regard to jurisdictional claims in published maps and institutional affiliations. 